Reprod. Nutr. Develop., 1988, 28 (3 B), 687-692

\title{
Plusieurs classes de myoblastes participent à la formation des muscles du squelette chez les oiseaux
}

\author{
V. MOULY, M. Y. FISZMAN
}

Unité de Biochimie, Département de Biologie Moléculaire, Institut Pasteur, 25, rue du Dr Roux, 75724 Paris Cedex 15.

Summary. Participation of several classes of myoblasts in the formation of skeleton muscles in birds.

Our results show that when myoblasts, isolated at different stages during muscle development, were cultured they formed myotubes expressing distinct phenotypes. An early phenotype was expressed by myoblasts isolated from 4-5 day old embryos and this phenotype could be modulated according to the culture conditions, i.e. seeding of the myoblasts as isolated cells or as reaggregated clumps of cells. An intermediate phenotype was expressed by myoblasts isolated from 7-8 day old embryos and it was independent of culture condition. A late phenotype was expressed by myoblasts isolated from embryos older than 9 days. In this case again, it could be modulated by culture conditions but, this time, modulation was brought about by subculturing the cells before they differentiate. These various phenotypes do not result from environmental differences in the culture but reflect the existence of distinct classes of myoblasts present at these different stages. This was demonstrated by isolating homogeneous clones of myoblasts at these stages and by showing that they express the corresponding phenotype.

\section{Introduction.}

Grâce à l'utilisation de cultures en conditions clonales, il a été possible de montrer que l'accroissement du pourcentage des myoblastes au cours du développement embryonnaire est un phénomène discontinu (Bonner et Hauschka, 1974). De plus les mêmes auteurs ont également montré que cette discontinuité était en fait reliée à l'apparition séquencielle de cellules musculaires présentant des exigences nutritionnelles distinctes. De tels résultats ont conduit ces auteurs à proposer l'existence d'une série de classes distinctes de myoblastes apparaissant de manière séquencielle au cours du développement des muscles embryonnaires. Toutefois, aucune donnée biochimique n'existant sur le sujet, il nous a paru intéressant de reprendre l'analyse de ce phénomène en essayant de caractériser biochimiquement ces différents myoblastes. Cette contribution a pour but de montrer qu'effectivement, des myoblastes isolés à des stades embryon- 
naires précis, expriment en culture des phénotypes spécifiques et distincts. De plus, l'utilisation de virus oncogènes nous a permis d'isoler des lignées stables de myoblastes correspondant à ces stades embryonnaires et de prouver ainsi que ce sont bien des classes distinctes de myoblastes qui sont présentes au cours du développement des muscles chez l'embryon.

\section{Matériel et méthodes.}

Culture et transformation des myoblastes. - Les myoblastes ont été isolés à partir des blastèmes de membres d'embryons de caille âgés de 4 à 9 jours et mis en culture selon un protocole décrit précédemment (Mouly et al., 1987). Les myoblastes réassociés ont été obtenus en suivant un protocole décrit par De Haan (McDonald et al., 1972). Pour transformer les myoblastes nous avons utilisé un mutant thermosensible du virus du sarcome de Rous (RSVtsNY68) ainsi qu'il est décrit dans Montarras et Fiszman (1983).

Analyse des protéines. - Les protéines synthétisées par les différents types cellulaires ont été analysées par la technique d'électrophorèse bidimensionnelle (O'Farrell, 1975) à partir d'extraits cellulaires totaux (Fiszman et al., 1980).

\section{Résultats.}

Différenciation des myoblastes isolés à différents stades embryonnaires.

Cas des embryons de 4 jours. - Quand on met en culture des cellules obtenues à partir des blastèmes de membre d'embryons de 4 jours on constate qu'une faible proportion d'entre elles sont capables de former des myotubes. Le pourcentage de fusion (\% des noyaux présents dans les myotubes) étant de l'ordre de 5 à $7 \%$. Ainsi qu'on peut le voir sur la figure 1A, ces myotubes synthétisent essentiellement deux chaînes légères de la myosine : LC1 F et LC2 S. Le fait d'utiliser du milieu conditionné ne change rien au phénotype exprimé, au contraire, les mêmes cellules maintenues sous forme de micro explants expriment un phénotype caractérisé par la présence de toutes les chaînes légères de la myosine (résultat non présenté). Ceci semblait donc indiquer qu'il était possible de moduler le phénotype exprimé par ces myoblastes différenciés. Afin de vérifier cette hypothèse, nous avons utilisé un procédé de réaggrégation et analysé le phénotype exprimé par ces cultures. Ainsi qu'on peut le voir sur la figure $1 \mathrm{~B}$, les myoblastes réassociés, une fois différenciés, synthétisent un mélange de chaînes légères de la myosine dans lequel LC1 $F$, LC2 S et LC2 $F$ sont les formes majoritaires et LC1 $S$ est présent sous forme de traces.

Cas des embryons de 6 jours. - Si on met en culture des cellules obtenues à partir des blastèmes d'embryons de 6 jours, on constate que la population de cellules myogéniques a augmenté puisque le pourcentage de fusion est d'environ 


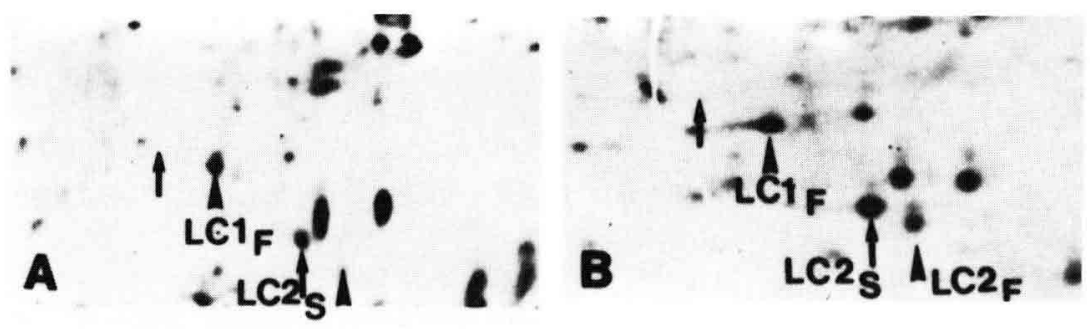

FIG. 1. - Chaînes légères de la myosine synthétisées par les cellules d'embryon de 4 jours. A) Cellules dissociées; B) Cellules réassociées. Les pointes de flèches indiquent les chaînes légères appartenant au phénotype rapide et les flèches celles appartenant au phénotype lent.

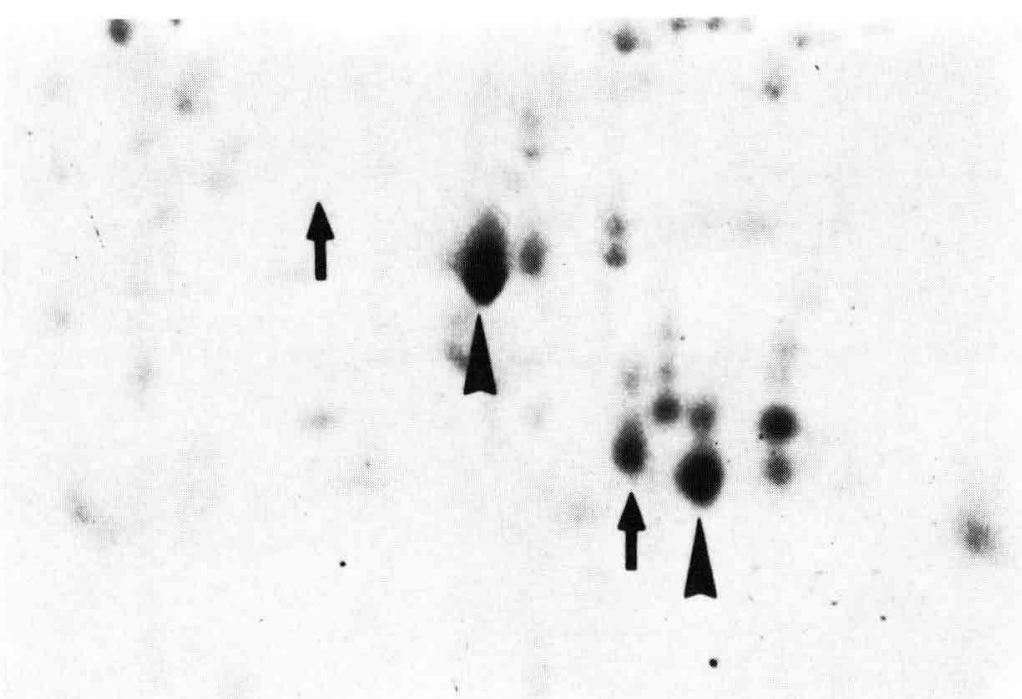

FIG. 2. - Chaines légères de la myosine synthétisées par les cellules d'embryon de 6 jours. Mêmes symboles que pour la figure 1 .

20 à $25 \%$. Quand on analyse le phénotype exprimé par ces myotubes on constate la présence des quatre chaînes légères de la myosine dans les mêmes proportions que celles observées avec les cellules précédentes réassociées (fig. 2). Comme on pouvait s'y attendre, le fait de faire se réassocier les cellules ne change rien au phénotype exprimé (résultat non présenté). II est d'ailleurs intéressant de noter que ce phénotype est exactement celui observé quand on analyse les fibres musculaires présentes dans le blastème à ce stade embryonnaire (Toutant $e t$ al., 1983).

Cas des embryons de 9 jours. - A ce stade embryonnaire, la majorité des cellules présentes sont des cellules musculaires puisque le pourcentage de fusion avoisine maintenant 75 à $80 \%$. Ces cultures expriment un phénotype différent des précédents puisque maintenant on note la présence de deux chaînes légères 
de la myosine : LC1 $\mathrm{F}$ et LC2 F (fig. 3A). Si on fait se réassocier les cellules avant qu'elles se différencient, on constate que le phénotype change puisque toutes les chaînes légères sont maintenant exprimées (fig. 3B). De même, si on oblige les myoblastes à se diviser pendant plusieurs cycles avant de se différencier (ceci peut être obtenu soit en sous-cultivant les cellules soit en les traitant de manière prolongée par des facteurs mitogènes), alors on retrouve également l'expression des quatre chaînes légères (fig. $3 \mathrm{C}$ ). II est important de noter que ce phénotype n'est toutefois pas identique à celui exprimé par les cellules d'embryons de 6 jours. En effet, ces dernières ne synthétisent que les formes isomorphes rapides des sous-unités de la tropomyosine alors que les cellules d'embryon de 9 jours, sous-cultivées, synthétisent en plus la forme isomorphe lente de la sous-unité $\alpha$ (fig. 4).

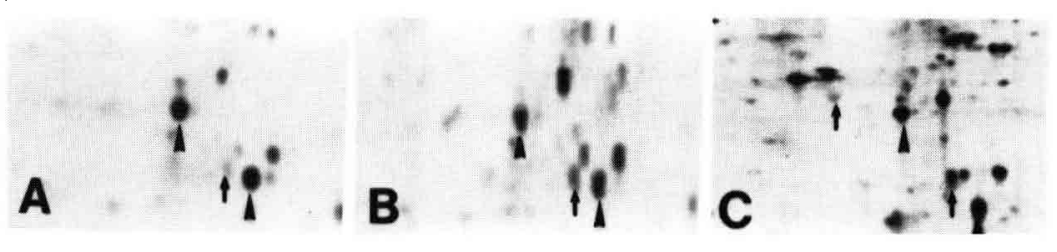

FIG. 3. - Chaînes légères de la myosine synthétisées par les cellules d'embryon de 9 jours. A) Cellules dissociées; B) Cellules réassociées; C) Cellules sous-cultivées. Mêmes symboles que pour la figure 1
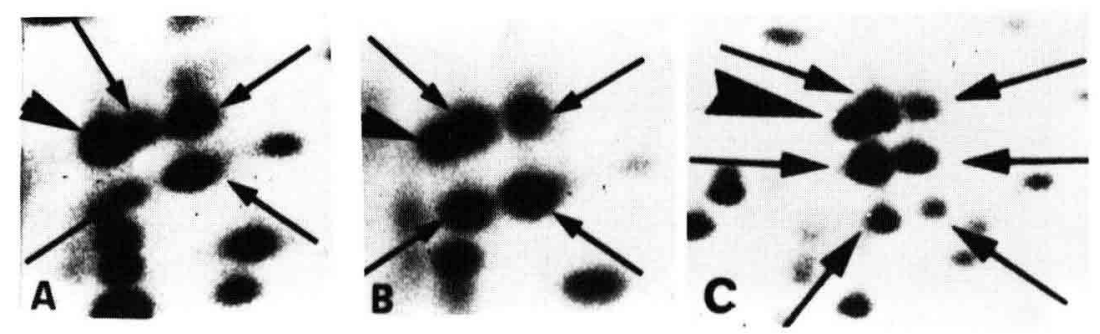

FIG. 4. - Tropomyosine synthétisée par les cellules d'embryon de 6 jours (A), les cellules d'embryons de 9 jours (B) et les cellules sous-cultivées d'embryon de 9 jours (C). Les flèches indiquent la position des tropomyosines musculaires et la pointe de flèche indique la position d'une protéine qui sert de référence.

Deux hypothèses peuvent rendre compte de nos résultats : 1) ces phénotypes correspondent à des conditions d'environnement différentes dans les cas de culture que nous avons analysés, ou 2) chaque stade embryonnaire est caractérisé par une population distincte de myoblastes. Pour distinguer entre ces deux hypothèses, nous avons cherché à isoler des populations homogènes de cellules musculaires à partir de chacun des stades embrynnaires et pour cela nous avons utilisé le RSVtsNY68.

\section{Phénotype exprimé par les différentes cellules musculaires transformées.}

Nous avons isolé des cellules myogéniques transformées soit à partir d'embryons de 4 jours, soit à partir d'embryons de 9 jours. La figure 5 présente 


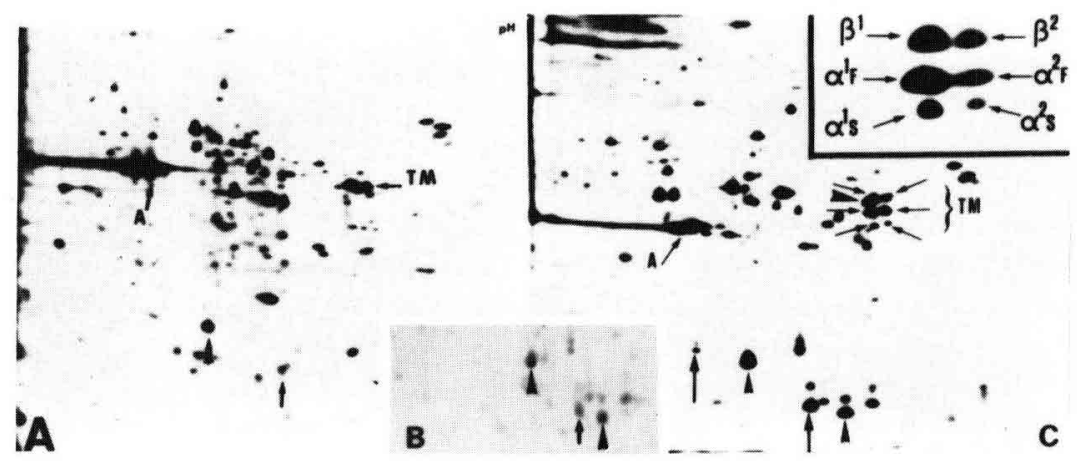

FIG. 5. - Protéines synthétisées par les clones transformés. A) Clone précoce en milieu normal: B) Clone précoce en présence d'extrait embryonnaire (région des chaînes légères); C) Clone tardif (l'encart présente la région des tropomyosines). Mêmes symboles que pour la figure 1.

l'analyse des protéines synthétisées par un représentant de chacun de ces groupes. Ainsi qu'on peut le voir chacun de ces clones exprime le phénotype spécifique du stade embryonnaire à partir duquel il a été isolé, qui plus est, le clone précoce exprime un phénotype modulable. Ainsi, le clone précoce synthétise LC1 $\mathrm{F}$ et LC2 $\mathrm{S}$ dans les conditions normales de culture (fig. 5A) mais synthétise les quatre chaînes légères si on ajoute au milieu de l'extrait embryonnaire (fig. 5B). En ce qui concerne le clone tardif, il exprime les quatre chaînes légères comme les myoblastes sous-cultivés (fig. $5 \mathrm{C}$ ). II faut cependant noter une anomalie en ce qui concerne la synthèse de la tropomyosine. En effet, le clone précoce ne synthétise que la sous-unité $\alpha$ et pas de sous-unité $\beta$ (fig. 5A). Ceci n'est pas une particularité de ce clone puisque tous les clones précoces que nous avons analysé présentent cette même caractéristique. Le clone tardif synthétise toutes les tropomyosines y compris la sous-unité $\alpha$ lente (fig. 5C).

\section{Discussion.}

Au cours de ce travail nous avons montré que les cellules musculaires expriment des phénotypes spécifiques du stade embryonnaire auquel elles ont été prélevées. Nous avons ainsi défini trois phénotypes : précoce (4-5 jours in ovo), intermédiaire (6-7 jours) et tardif (au-delà de 8 jours). Le phénotype précoce est modulable et dépend des conditions de culture et en particulier d'interaction entre les cellules. Le phénotype intermédiaire est constant et quant au phénotype tardif il varie en fonction du temps pendant lequel les myoblastes se sont multipliés avant de se différencier. L'utilisation d'un mutant thermosensible du virus du sarcome de Rous nous a permis d'isoler des clones de cellules myogéniques transformées à partir des populations cellulaires prélevées aux différents stades embryonnaires. Nous pouvons alors montrer que les différents clones transformés expriment le phénotype correspondant au stade de l'isolement des cellules. Ainsi donc, les phénotypes que nous avons définis traduisent bien l'existence de classes distinctes de myoblastes apparaissant séquentiellement au cours du développement. 
Quelle hypothèse peut-on formuler quant à la fonction de ces différentes populations. Les études histochimiques ont montré qu'il est possible de définir deux types de fibres musculaires: les fibres de première génération et les fibres secondaires. Sur la base de la cinétique d'apparition de ces différentes fibres (McLennan, 1983), il est tentant de proposer que les myoblastes précoces sont les cellules précurseurs des fibres de première génération alors que les myoblastes tardifs seraient responsables de l'apparition des fibres secondaires qui vont former la masse des fibres musculaires et qui se spécialiseront en muscles rapides et lents en fonction du type d'innervation ou d'autres paramètres encore à définir. II est intéressant de noter qu'une hypothèse semblable a été formulée par le groupe de Stockdale sur la base d'études concernant les chaînes lourdes de la myosine (Miller et Stockdale, $1986 a$ et b).

$13^{e}$ Réunion du groupe Développement I.N.R.A., Cap d'Agde, 25-27 mai 1987.

Remerciements. - Ce travail a bénéficié d'aide financière du CNRS, de l'INSERM, du CEA, du Ministère de l'Industrie et de la Recherche, de la Fondation pour la Recherche Médicale Française, de I'ADRC, de la Ligue Française contre le Cancer, de l'Association des Myopathes de France et de la Muscular Dystrophy Association of America.

\section{References}

BONNER P. H., HAUSCHKA S. D., 1974. Clonal analysis of vertebrate myogenesis. I. Early developmental events in the chick limb. Dev. Biol., 37, 317-328.

FISZMAN M. Y., MONTARRAS D., WRIGHT W., GROS F., 1980. Expression of myogenic differentiation and myotube formation by chick embryo myoblasts in the presence of sodium butyrate. Exp. Cell Res., 126, 31-37.

McDONALD T. F., SACHS H. G., DE HAAN R. L., 1972. Development of sensitivity to tetrodotoxin in beating chick embryo hearts, single cells and aggregates. Science, 176, 1248-1250.

McLENNAN I. S., 1983. Differentiation of muscle fiber type in the chicken hind limb. Dev. Biol., 97. 222-228.

MILLER J. B., STOCKDALE F. E., 1986a. Developmental origin of skeletal muscle fibers: clonal analysis of myogenic cell lineages based on fast and slow myosin heavy chain expression. Proc. natl. Acad. Sci. USA, 83, 3860-3864.

MILLER J. B., STOCKDALE F. E., 1986b. Developmental regulation of the multiple myogenic cell lineages of the avian embryo. J. Cell Biol., 103, 2197-2208.

MONTARRAS D., FISZMAN M. Y., 1983. A new phenotype is expressed by subcultured quail myoblasts isolated from future fast and slow muscles. J. biol. Chem., 258, 3883-3888.

MOULY V., TOUTANT M., FISZMAN M. Y., 1987. Chick and quail limb bud myoblasts, isolated at different times during muscle development, express stage-specific phenotypes when differentiated in culture. Cell Differ., 20, 17-25.

O'FARRELL P. H., 1975. High resolution two-dimensional electrophoresis of proteins. J. biol. Chem., 230, 4007-4021.

TOUTANT M., TOUTANT J. P., MONTARRAS D., FISZMAN M. Y., 1983. Potential phasic and tonic muscles express a common set of fast and slow myosin light chains and fast tropomyosin during early development of chick embryo. Biochimie, 65, 637-642. 